\title{
Brachiocephalic Vein Aneurysm Treated with Surgery: A Case Report
}

\section{Jin Hong Wi}

Department of Thoracic and Cardiovascular Surgery, Busan Paik Hospital, Inje University College of Medicine, Busan, South Korea.

\section{ABSTRACT}

Brachiocephalic vein aneurysm is an extremely rare condition of unknown origin. It may be asymptomatic, presenting as an incidental finding or as a result of complications it causes. We report a case of a 54-year-old man who was asymptomatic and diagnosed with an isolated saccular aneurysm of the left brachiocephalic vein using contrast-enhanced computed tomography. The patient underwent aneurysmectomy through a median sternotomy with no requirement for a vascular graft or cardiopulmonary bypass. The patient had an uneventful postoperative course and remained well two months after the surgery.

\section{INTRODUCTION}

Aneurysm arising from the thoracic veins is very rare. When present, fusiform dilatation of the superior vena cava is most commonly seen [Haniuda 2000; Bansal 2010; Taira 1981]. Since brachiocephalic vein aneurysm was first reported in 1928 [Harris 1928], only about 20 cases have been reported in the literature. Moreover, a few of them have discussed its surgical treatment [Galvaing 2018; Huang 2017; Aggarwal 2017; Lohrenz 2018; Nakada 2015; Akibaa 2012; Sakai 2011; Bartline 2016; Hosein 2007; Sayed 2013]. We report a case of a patient who had an isolate brachiocephalic vein aneurysm and underwent surgery successfully without cardiopulmonary bypass or vascular graft.

\section{CASE REPORT}

A 54-year-old asymptomatic man was referred to our department owing to a large mediastinal mass that was incidentally discovered on computed tomography (CT) without intravenous contrast. The patient was asymptomatic and did not report any underlying disease or local trauma such as clavicular or rib fracture. There was no history of surgical intervention or central venous cannulation of the neck or upper extremities performed in the past. The chest $\mathrm{X}$-ray revealed no abnormal findings, and inflammatory markers were normal. A

Received fune 6, 2019; accepted fune 27, 2019.

Correspondence: Fin Hong Wi, Department of Thoracic and Cardiovascular Surgery, Busan Paik Hospital, Inje University College of Medicine, 75 Bokjiro, Busanjin-gu, Busan 614-735, Korea; 82-51-890-6834; fax: 82-51-8911297 (e-mail:wiccy@hanmail.net). contrast-enhanced CT revealed a large saccular dilatation of the left brachiocephalic vein (Figure 1). The aneurysm measured $62 \mathrm{~mm} \times 38 \mathrm{~mm}$. There were no filling defects to suggest thrombosis within the aneurysmal sac, and pulmonary embolism was not detected. The right brachiocephalic vein, subclavian veins, and superior vena cava were normal. Transthoracic echocardiography showed that both contractility and diastolic functions were normal, and intracardiac anomaly was absent.

Surgery was proposed because of the risk of embolic events or rupture. The patient also actively wanted to undergo an operation to avoid such complications. Moreover, as the patient was healthy and young, it was expected that the surgical risk would be very low.

A median sternotomy was performed. We first controlled the proximal and distal brachiocephalic vein to prevent excessive blood loss in case of hemorrhage. The aneurysm carefully was dissected (Figure 2A), and its wide base identified. It subsequently was opened longitudinally after cross-clamping of its base, taking great care to not narrow the left brachiocephalic vein. Complete excision of the aneurysm was performed, leaving a $3.0-\mathrm{cm}$ defect in the underside of the brachiocephalic vein. The wall of the aneurysm was very thin and friable. There was no thrombus or phlebolith within the lesion (Figure 2B). The defect in the underside of the vein was repaired with continuous suture using 5-0 nonabsorbable monofilament suture (Figure 3).

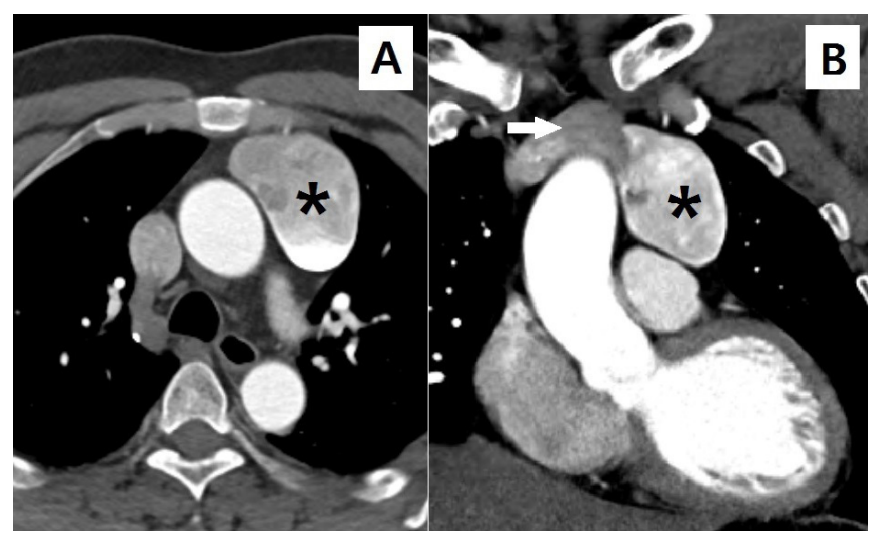

Figure 1. Contrast enhanced computed tomography. A. Cross-sectional imaging, well-circumscribed anterior mediastinal mass immediately antero-lateral to the ascending aorta, showing intense contrast enhancement without filling defects (size of mass: $62 \mathrm{~mm} \times 38 \mathrm{~mm}$ ), B. Coronal plane imaging, aneurysm was seen in continuity with the left brachiocephalic vein (black asterisk: brachiocephalic vein aneurysm, white arrow: normal left brachiocephalic vein). 

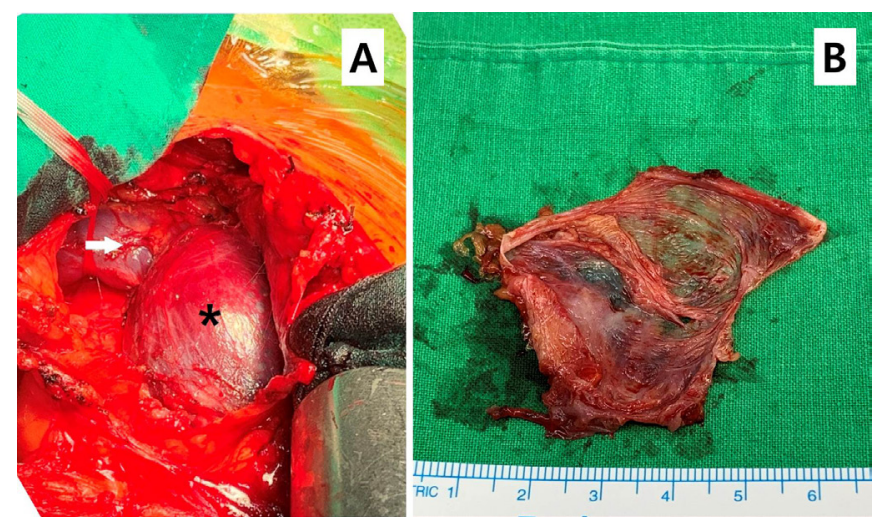

Figure 2. A. Aneurysm and brachiocephalic vein, which was carefully dissected (black asterisk: brachiocephalic vein aneurysm, white arrow: normal left brachiocephalic vein), B. The wall of aneurysm, which was very thin and friable (no thrombus or phlebolith formation within aneurysmal sac).

Pathological examination of the aneurysm revealed no abnormality. The sampled tissue was made of a normal venous structure, making it a true aneurysm. The patient had an uneventful postoperative course and was discharged home on the postoperative day 4. He remained well two months after the surgery. A contrast-enhanced CT of his chest was obtained that showed the left brachiocephalic vein with normal flow.

\section{DISCUSSION}

Brachiocephalic vein aneurysm is a rare condition, usually detected as incidental findings. Very little is known about the etiology and natural history of these vascular lesions. They can be congenital in origin or acquired secondary to trauma, inflammation, or degenerative change in the vessel wall [Haniuda 2000; Schatz 1962; Güney 2004]. The etiologic factors of the aneurysm in our patient remain unclear. Congenital malformation might be likely the cause of the aneurysm in this case, because there is no evidence of inflammation, trauma, degenerations, or neoplasms. If there is the case of solitary saccular aneurysms without symptoms and hemodynamically significant narrowing proximal to the aneurysm, a congenital etiology appears most likely [Haniuda 2000].

Most patients with brachiocephalic vein aneurysm are asymptomatic, and the lesion is incidentally detected as a mediastinal mass on chest radiography or CT as in this patient. Aneurysms can cause chest pain, dyspnea, and recurrent nerve paralysis because of compression of continuous structures [Bansal 2010, Aggarwal 2017; Lohrenz 2018; Nakada 2015; Hosein 2007; Hosaka 2011; Buehler 2013]. They may sometimes be associated with a rupture or pulmonary embolism [Taira 1981; Ream 1972; Schild 1992; Taira 1981].

Cross-sectional imaging, using either magnetic resonance imaging or CT, is sufficient for accurately diagnosing venous and arterial aneurysms and enabling planning of the surgical strategy [Galvaing 2018; Aggarwal 2017; Gozdziuk 2004].

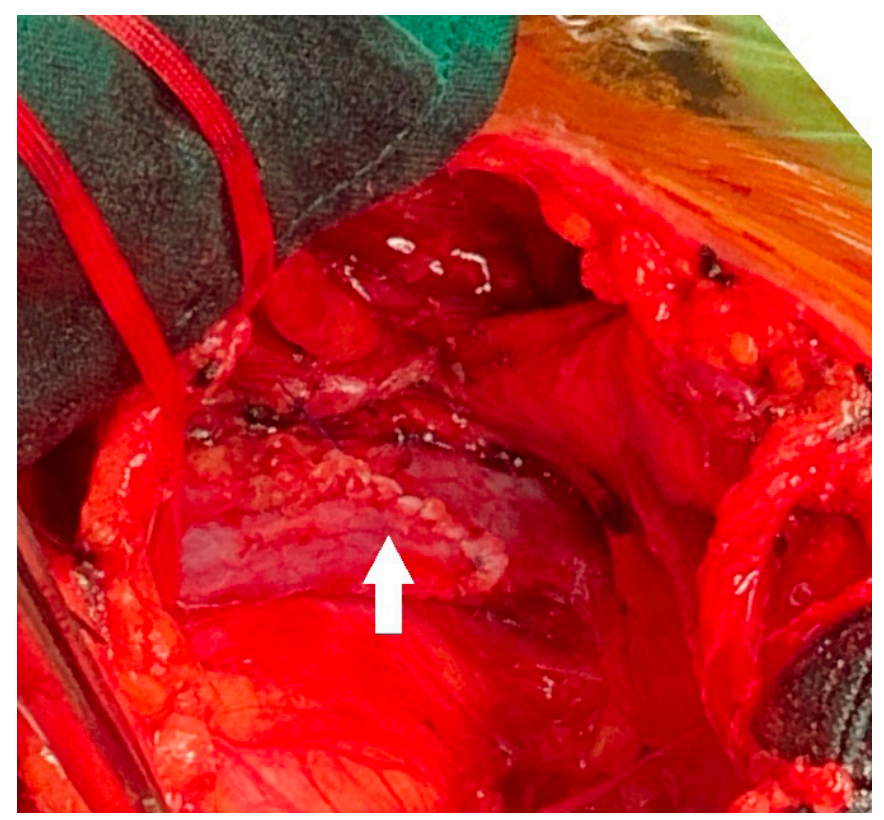

Figure 3. White arrow: left brachiocephalic vein after aneurysmectomy and primary repair of the defect in the underside of the vein.

In recent years, the use of multiplanar CT reformations to demonstrate the anatomic continuity of the aneurysms with the thoracic venous system has obviated the need for invasive venous angiography [Dua 2011]. Intravenous contrast plays a vital role in the diagnosis of venous aneurysms and helps to detect or rule out complications such as rupture and thrombosis within the aneurysm. This information is vital in deciding the treatment approach and planning surgical strategy if open-repair is indicated [Buehler 2013; Dua 2011]. With contrast-enhanced CT, we could diagnose and plan the surgical strategy without difficulty.

Treatment options are controversial and not well defined because the risks of pulmonary embolism, rupture, and venous obstruction are unknown. It is suggested that saccular or symptomatic aneurysms surgically can be repaired to avoid complications, whereas asymptomatic fusiform or small-sized aneurysms safely can be observed [Galvaing 2018; Hosein 2007; Güney 2004; Hosaka 2011; Buehler 2013; Dua 2011]. Endovascular interventions that involve coil embolization of the aneurysm and stent implantation have been proposed as treatment options in case of subclavian vein aneurysm [San 2010], but they have not been described so far in case of brachiocephalic vein aneurysm.

As for surgical approaches, median sternotomy is preferred over video-assisted thoracic surgery because of the possible intraoperative challenges such as pulmonary embolism, massive bleeding from tearing of the thin venous wall, and reconstruction of brachiocephalic vein [Huang 2017]. However, literature research shows few cases of successful surgical approach other than median sternotomy, such as thoracotomy [Sayed 2013] or video-assisted thoracoscopy [Lohrenz 2018]. Cardiopulmonary bypass (CPB) frequently is reported to be necessary to allow safe mobilization and resection of 
aneurysm [Aggarwal 2017; Bartline 2016; Hosein 2007]. However, in our patient, we believed that we could obtain the same safety with gentle dissection of the aneurysm and rapid control of the left brachiocephalic vein without any adverse events. If possible, in selected cases such as this patient, it is desirable to perform surgery without $\mathrm{CPB}$ to reduce the mortality and morbidities. To our knowledge, there have been only four reported cases of surgery for isolated brachiocephalic vein aneurysm without CPB [Huang 2017; Lohrenz 2018; Sakai 2011; Sayed 2013].

According to previous reports, the prognosis after successful resection is very good [Galvaing 2018; Huang 2017; Aggarwal 2017; Lohrenz 2018; Nakada 2015; Akibaa 2012; Sakai 2011; Hosein 2007; Sayed 2013]. There are no reported cases of recurrence or of the development of similar lesions elsewhere.

\section{REFERENCES}

Aggarwal R. Gautam R, Jhamb D, Sivasankar R. 2017. Diagnosing thoracic venous aneurysm: A contemporary imaging perspective. Indian Journal of Radiology and Imaging 27(3):350-353.

Akibaa T, Morikawab T, Hirayamaa S, Ohki T. 2012. Thymic haemangioma presenting with a left innominate vein aneurysm: insight into the aetiology. Interactive CardioVascular and Thoracic Surgery 15: 925-927.

Bansal K, Deshmukh H, Popat B, Rathod K. 2010. Isolated left brachiocephalic vein aneurysm presenting as a symptomatic mediastinal mass. Journal of Medical Imaging and Radiation Oncology 54:462-464.

Bartline PB, McKellar SH, Kinikini DV. 2016. Resection of a Large Innominate Vein Aneurysm in a Patient with Neurofibromatosis Type 1. Ann Vasc Surg 30:157.e1-157.e5.

Buehler MA, Ebrahim FS, Popa TO. 2013. Left Innominate Vein Aneurysm: Diagnostic Imaging and Pitfalls. Int J Angiol 22:127-130.

Dua SG, Kulkarni AV, Purandare NC, Kulkarni S. 2011. Isolated left innominate vein aneurysm: A rare cause of mediastinal widening. Journal of Postgraduate Medicine 57(1):40-41.

Galvaing G, Gaudin M, Medous MT, Filaire M, Ferrand C, Vichy. 2018. Left Brachiocephalic Venous Aneurysm: A Rare Clinical Finding Ann Vasc Surg 48:253.e5-253.e6.

Gozdziuk K, Czekajska-Chehab E, Wrona A, Tomaszewski A, Drop A. 2004. Saccular aneurysm of the superior vena cava detected by computed tomography and successfully treated with surgery. Ann Thorac Surg 78:e94 -5.
Güney B, Demirpolat G, Savas, R, Alper H. 2004. An unusual cause of mediastinal widening: bilateral innominate vein aneurysms. Acta Radiol $45: 266-268$

Haniuda M, Numanami H, Makiuchi A, Yamanda T, Imai Y, Sone S. 2000. Solitary Aneurysm of the Innominate Vein. Journal of Thoracic Imaging 15:205-207.

Harris RI. 1928. Congenital venous cyst of the mediastinum. Ann Surg 88:953-956.

Hosaka A, Kato M, Kato I, Isshiki S, Okubo N. 2011. Brachiocephalic venous aneurysm with unusual clinical observations. J Vasc Surg 54:77S-9S.

Hosein RB, Butler K, Jones T, Brawn WJ, Barron DJ. 2007. Innominate Venous Aneurysm Presenting as a Rapidly Expanding Mediastinal Mass. Ann Thorac Surg 84:640 -2.

Huang W, Jiang GN. 2017. A rare case of left innominate vein aneurysm mimicking thymoma. Interactive Cardiovascular and Thoracic Surgery 25:669-670.

Lohrenz C, Ruckner D, Wintzer O, Fengels WG, Meierling S. 2018. Large left innominate vein aneurysm presenting as an anterior mediastinal tumor in a young female. Vasa 47(6):515-517.

Nakada T, Akiba T, Inagaki T, Morikawa T, Ohki T. 2015. Thymic Cavernous Hemangioma With a Left Innominate Vein Aneurysm. Ann Thorac Surg 100:320-2.

Ream CR, Giardina A. 1972. Congenital superior vena cava aneurysm with complications caused infectious mononucleosis. Chest 62:755-7.

Sakai M, Kanzaki R, Kozuka T, et al. 2011. Left Innominate Venous Aneurysm Presenting as an Anterior Mediastinal Mass. Ann Thorac Surg 91:1995.

San NEM, Gutierrez VM, Revilla A, Vaquero C. 2010. Subclavian venous aneurysm: endovascular treatment. J Vasc Intervent Radiol 21:1306-8.

Sayed SA, Sahu D, Khandekar J, Jadhav U. 2013. Large aneurysm of innominate vein: Extremely rare cause of meidastinal mass. Indian J Thorac Cardiovasc Sirg 26:198-9.

Schatz IJ, Fine G. 1962. Venous aneurysms. N Engl J Med 266:1310-2.

Schild H, Berg S, Weber W, Schmied W, Steegmuller KW. 1992. The venous aneurysm. Aktuelle Radiol 2:75-80.

Taira A, Akita H. 1981. Ruptured venous aneurysm of the persistent left superior vena cava. Angiology 32:656-659.

Taira A, Akita H. 1981. Ruptured venous aneurysm of the persistent left superior vena cava. Angiology 32:656-9. 